\title{
Chimioprophylaxie antitrypanosomienne de bovins N'Dama importés de Sénégambie J.P. Dehoux 1 et du Zaïre au Gabon
}

DEHOUX (J.P.). Chimioprophylaxie antitrypanosomienne de bovins N'Dama importés de Sénégambie et du Zaïre au Gabon. Revue Élev. Méd. vét. Pays trop., 1990, 43 (3): 337-341

Un programme de chimioprophylaxie antitrypanosomienne, associant l'acéturate de diminazène et le chlorure d'isométamidium, a été élaboré pour des bovins de race N'Dama nouvellement importés du Sénégal, de Gambie et du Zaïre car le transfert de ces animaux entraînait une défaillance de leur trypanotolérance. Mots clés : Bovin N'Dama - Importation - Trypanocide - Prophylaxie - Trypanotolérance - Gabon.

\section{INTRODUCTION}

Le Gabon à dû importer du bétail N'Dama en provenance de plusieurs pays (Sénégambie et Zaïre) pour développer et intensifier un élevage bovin. Le ranch de la Ngounié a accueilli 2467 bovins (1668 de Sénégambie et 799 du Zaïre) importés entre 1984 et 1987.

Confrontés aux glossines et à la trypanosomose, les bovins sont protégés par une chimioprophylaxie antitrypanosomienne. Cette étude reprend le programme d'utilisation des trypanocides et certains aspects de l'adaptation du bétail dans une nouvelle région.

\section{MATÉRIEL ET MÉTHODE}

\section{Lieu d'étude}

Le ranch de la Ngounié est situé dans le sud du Gabon, à $30 \mathrm{~km}$ de la ville de Ndendé. Les savanes sont dominées par une association d'Hypparrhenia diplandra et de Loudetia arundinacea. La région jouit d'un climat forestier guinéen avec une pluviométrie annuelle de $1500 \mathrm{~mm}$. La saison sèche s'étend de juin à octobre.

Le relief du ranch est émaillé de nombreux massifs et galeries forestières, refuges de Glossina palpalis palpalis et de Glossina fusca tabaniformis.

1. 59 rue Grande, 5141 Andoy-Wierde, Belgique.

Reçu le 13.2.1990, accepté le 5.3.1990.

\begin{abstract}
Animaux
Ils sont tous de race N'Dama. Les animaux importés en $1986(521)$ et en $1987(1100)$ ont été protégés suivant le protocole décrit ci-après, suite aux problèmes pathologiques observés sur le lot de 399 bovins importés en 1985, qui n'avait pas fait l'objet, immédiatement, d'une couverture antitrypanosomienne. Ce dernier lot, considéré comme le lot témoin, est arrivé dans les mêmes conditions de transport, à la même période de l'année et a été placé sur la même pâture de mise en quarantaine que les animaux de 1986 et 1987.
\end{abstract}

Sans reposer sur un protocole expérimental précis, cette étude donne néanmoins des indications importantes sur la nécessité de protéger le bétail nouvellement importé contre les risques de trypanosomose.

Les animaux arrivent au mois de novembre, moment du retour en force des pluies.

\section{Trypanocides}

Deux produits sont utilisés, l'acéturate de diminazène (Bérénil R) dosé à $3,5 \mathrm{mg} / \mathrm{kg}$ de poids vif (5) et le chlorure d'isométamidium (Trypamidium R) utilisé à la dose prophylactique minimale de $0,5 \mathrm{mg} / \mathrm{kg}$ de poids vif (12).

Ces deux médicaments sont injectés par voie intramusculaire dans la région cervicale.

\section{Protocole d'administration des trypanocides}

Selon le temps de présence des animaux sur le ranch et selon leur âge, différents protocoles d'administration des trypanocides sont instaurés.

Le bétail importé, débarqué des bétaillères, est soumis immédiatement à une injection d'acéturate de diminazène, suivie, une semaine après au plus tard, d'une administration de chlorure d'isométamidium.

Le bétail adulte présent sur le ranch depuis plus de 6 mois est ensuite soumis, deux fois par an, au même traitement trypanocide; une première fois au mois de juin (en fin de saison des pluies) et une seconde fois au mois d'octobre (en fin de saison sèche). 
Les veaux reçoivent, entre la deuxième et la troisième semaine de leur existence, une injection d'acéturate de diminazène. Cette période coîncide avec le marquage et l'écornage de ces animaux.

Les sevrons suivent le même protocole que celui instauré pour les adultes importés.

\section{Analyses de sang}

Des prélèvements de sang ont été effectués au moment de l'arrivée du bétail en 1986 (98 bêtes) et en 1987 (57), pour une détection de parasitémie, et bimensuellement sur un lot de 50 animaux soumis au traitement prophylactique en 1986, pour apprécier la durée de protection des trypanocides et le taux mensuel de parasitémie détectée.

Le sang est prélevé sur une veine marginale de l'oreille. Des examens à frais, des frottis et des microhématocrites ont été analysés.

\section{Appréciation de la trypanotolérance}

Deux lots $(A$ et $B)$ de 30 animaux ont été constitués au cours de la troisième année d'adaptation d'un troupeau de N'Dama sénégalaises importées en 1986. Ces deux lots ont été mis sous couverture trypanocide selon le protocole décrit plus haut en début de test (juin). Le lot $\mathrm{A}$ a été remis sous prophylaxie antitrypanosomienne en fin de saison sèche (octobre) tandis que le lot $\mathrm{B}$ continuait à évoluer sans protection.

Les animaux des deux lots ont également été pesés en début de test, puis bimensuellement pendant toute la durée de celui-ci. De même pour la détection de la parasitémie, qui fut contrôlée tous les mois.

\section{RÉSULTATS}

Le tableau I reprend les taux annuels de mortalité des bovins adultes en fonction de leur temps de présence au ranch et de leur année d'importation.

TABLEAU I Taux annuels de mortalité (p. 100) des bovins N'Dama adultes importés au Gabon, selon le nombre d'années de présence sur le ranch de la Ngounié.

\begin{tabular}{|c|c|c|c|c|}
\hline $\begin{array}{c}\text { Année } \\
\text { d'importation * }\end{array}$ & 1re année & $2^{e}$ année & $3^{\theta}$ année & $4^{e}$ année \\
\hline $\begin{array}{l}1985 \\
1986 \\
1987\end{array}$ & $\begin{array}{r}19,8 \\
3,3 \\
2,9\end{array}$ & $\begin{array}{l}3,3 \\
3,1 \\
2,7\end{array}$ & $\begin{array}{l}2,5 \\
2,7\end{array}$ & 2,1 \\
\hline
\end{tabular}

* Les bovins arrivent au mois de novembre, le taux de mortalité est calculé sur 12 mois lde novembre d'une année à octobre de l'année suivante).
Les animaux importés en 1985 ont connu une flambée de mortalités au cours du deuxième mois de leur présence sur le ranch (vers le $40 \mathrm{e}$ jour). Imputables à la trypanosomose, à la cowdriose et à l'anaplasmose dans des proportions non définies, ces morts sont survenues brusquement sur du bétail qui récupérait de son voyage dans de bonnes conditions. Des administrations de trypanocides (acéturate de diminazène et de chlorure d'isométamidium) et d'antibiotiques (oxytétracycline LA) ont permis de juguler ces accès de mortalité ( 70 bovins, soit $17,5 \mathrm{p} .100)$ dans un intervalle de 10 jours. Suite à ces observations, inconnues en 1984, une couverture antitrypanosomienne énergique a été instaurée lors des importations suivantes. Une différence statistiquement significative a été observée quant au taux de mortalité, calculé pour 12 mois, entre les animaux arrivés en 1985, d'une part, et les animaux de 1986 et 1987 d'autre part (tabl. I).

On remarque que 88,6 p. 100 des morts survenues chez le bétail importé en 1985 (pour 12 mois de présence sur le ranch) sont causés par les trois maladies suivantes: trypanosomose, cowdriose et anaplasmose, contre 16,1 p. 100 pour l'année 1987 (tabl. II). Pour cette même année, les cas de trypanosomose mortelle sont enregistrés chez des taureaux qui, en raison de leur comportement agressif, n'ont pu être examinés et traités lors de séances hebdomadaires de soins.

TABLEAU II Mortalité pour les 12 premiers mois de présence sur le ranch chez les animaux importés en 1985 et en 1987.

\begin{tabular}{|l|c|c|}
\hline \multicolumn{1}{|c|}{$\begin{array}{c}\text { Causes } \\
\text { de la mortalité }\end{array}$} & $\begin{array}{c}\text { Importations } \\
\text { de } 1985\end{array}$ & $\begin{array}{c}\text { Importations } \\
\text { de 1987 }\end{array}$ \\
\hline $\begin{array}{l}\text { Trypanosomose } \\
\text { Cowdriose }\end{array}$ & 70 & 3 \\
Anaplasmose & - & 1 \\
Tuberculose & 1 & 1 \\
Fracture & 2 & 2 \\
Phlegmons interdigités & - & 5 \\
Polyarthrite & - & 1 \\
Métrite & 1 & 2 \\
Luxation coxofémorale & 1 & 1 \\
Écrasement & 1 & 1 \\
Enlisement & - & 1 \\
Dystocie & - & 2 \\
Mort subite & - & 2 \\
Entérite aiguë & - & 1 \\
Torticolis & - & 1 \\
Épuisement & 1 & 3 \\
Plaies & 2 & 31 \\
Autres & 79 & \\
\hline Total & & \\
\hline
\end{tabular}

Le tableau III représente le nombre d'animaux sénégambiens détectés porteurs de trypanosomes lors de 
TABLEAU III Fréquence de la parasitémie détectée due aux trois espèces de trypanosomes chez les animaux importés en 1986 et en 1987.

\begin{tabular}{|c|c|c|c|c|}
\hline $\begin{array}{c}\text { Année } \\
\text { d'importation }\end{array}$ & $\begin{array}{c}\text { Nombre } \\
\text { d'animaux } \\
\text { détectés }\end{array}$ & $\begin{array}{l}\text { T. con- } \\
\text { golense }\end{array}$ & $T$. vivax & T. brucei \\
\hline $\begin{array}{l}1986 \\
1987\end{array}$ & $\begin{array}{c}25 / 98 \\
(25,5 \text { p. } 100) \\
15 / 57 \\
(26,3 \text { p. } 100)\end{array}$ & $\begin{array}{l}15 \\
10\end{array}$ & $\begin{array}{r}10 \\
5\end{array}$ & $\begin{array}{l}0 \\
0\end{array}$ \\
\hline
\end{tabular}

leur importation en 1986 et 1987, ainsi que le pourcentage d'infection due aux trois espèces de trypanosomes. Aucune différence significative n'a été observée entre les deux années. Les animaux issus du Zaïre étaient exempts de trypanosomes.

Huit animaux sur les 50 bovins examinés (16 p. 100) bimensuellement ont eu une parasitémie détectable après 45 jours de protection trypanocide, puis le taux de détection mensuel est passé à $4 \mathrm{p} .100$ (deux nouveaux cas par mois). Ces animaux commençaient à présenter des signes cliniques de la maladie au bout du quatrième mois de parasitémie.

Quatre animaux malades et trypanosés du lot B sont morts 6 mois après le début du test. Ces bovins ont eu une parasitémie détectée dès la fin de la protection trypanocide ; leur état sanitaire s'est dégradé dès leur deuxième mois de parasitémie. Comme d'autres animaux parasitémiques commençaient à dépérir également, le test a été interrompu et les animaux ont été placés sous couverture trypanocide. A ce moment, une différence significative quant à la comparaison des moyennes pondérales des deux lots était observée ( $t=1,73$ pour $\alpha=0,05$ ), tandis que 14 animaux sur les 26 survivants du lot $B$ avaient une parasitémie détectée (tabl. IV).

TABLEAU IV Évolution pandérale chez les animaux importés en 1986, soumis ou non à une prophylaxie antitrypanosomienne au cours de leur troisième année d'adaptation (moyenne des pesées en $\mathrm{kg}$ et écart-type).

\begin{tabular}{|c|c|c|c|c|}
\hline & Juin & Août & Octobre & Décembre \\
\hline Lot A & 248,1 & 252,0 & 239,9 & 250,4 \\
(traité) & $(27,1)$ & $(25,5)$ & $(19,2)$ & $(22,8)$ \\
Lot B & 244,5 & 243,7 & 233,9 & 234,1 \\
(non traité) & $(25,5)$ & $(21,3)$ & $(25,3)$ & $(25,9)$ \\
\hline $\begin{array}{c}\text { Comparaison } \\
\text { de moyennes * }\end{array}$ & NS & NS & NS & S \\
\hline
\end{tabular}

Lot $A: n=30 ;$ lot $B: n=26$ (en décembre).

- Au test t de Student, NS = différence non significative, $S=$ différence significative au seuil de 0,05 .

\section{DISCUSSION ET CONCLUSION}

La trypanotolérance est un caractère qui est loin d'être absolu. De nombreux facteurs de stress comme l'insuffisance alimentaire, un travail excessif, une verminose importante, une maladie infectieuse ou une adaptation à une nouvelle région, diminuent, voire inhibent, la trypanotolérance $(9,10,15)$.

Le bétail qui arrive au ranch est débilité par plusieurs jours de voyage long et pénible. Le milieu dans lequel il arrive est nouveau, le début de la saison des pluies coïncide avec une explosion démographique des populations de glossines et de tabanidés. La crise d'adaptation est réelle pour ces animaux. Les taux de mortalité, de morbidité et de natalité (15,5 p. 100 en première année) s'en ressentent. En 1985, on a cons taté une défaillance importante de la trypanotolérance qui a nécessité l'utilisation de trypanocides et d'antibiotiques pour enrayer les flambées de mortalité. C'est pourquoi, en 1986 et 1987, les animaux ont été protégés dès leur arrivée par l'administration de deux trypanocides, l'un curatif (l'acéturate de diminazène) qui va éliminer les trypanosomes existants et favoriser l'installation d'un autre médicament (le chlorure d'isométamidium), qui va protéger l'animal pendant une longue période $(2,5)$.

Au cours de leur adaptation, au rythme de deux injections annuelles, les animaux seront couverts chimiquement pendant plus ou moins cinq mois. On compte que, durant les sept mois pendant lesquels aucune couverture médicamenteuse n'existe, les attaques trypanosomiennes réveillent, et/ou adaptent au nouveau milieu la trypanotolérance. Les souches de trypanosomes n'étant pas les mêmes d'une région à l'autre, il faut un délai pour que les animaux élaborent de nouveaux types d'anticorps.

L'utilisation judicieuse de ces deux trypanocides atténue le risque d'apparition de résistance $(1,11)$. Elle permettrait le développement d'un seuil de tolérance aux trypanosomes, qui se traduirait par une augmentation de l'intervalle nécessaire entre deux traitements et par une amélioration des résultats zootechniques (2, 7, 17).

Une protection prophylactique durant toute l'année est contre-indiquée car le chlorure d'isométamidium ne permet pas au bétail de développer, pendant la période de protection, des réactions immunitaires à l'encontre des trypanosomes, du moins dans des conditions naturelles $(8,16)$.

On administre également aux veaux de l'acéturate de diminazène pour les aider à surmonter la crise de primo-infection. Les trypanocides agiraient en freinant l'incidence de la trypanosomose à mesure que le corps élimine le médicament. Le nombre de trypano- 


\section{J.P. Dehoux}

somes s'accroît, ce qui permet l'installation progressive de l'immunité $(3,14)$.

La récupération, au terme de la troisième année de présence sur le ranch, de taux de natalité $(65,8$ p. 100) et de mortalité semblables à ceux obtenus dans leur pays d'origine est un indice positif de leur adaptation (13).

Comme le montre le test d'appréciation de la trypanotolérance, au stade actuel de leur adaptation, les

DEHOUX (J.P.). Chemoprophylaxis of bovine trypanotolerance in N'Dama cattle imported from the Senegambia and from Zaire into Gabon. Revue Elev. Méd. vét. Pays trop., 1990, 43 (3) : 337-341

A programme of chemoprophylaxis against trypanosomiasis has been work out for the protection of newly imported N'Dama cattle from the Gambia, the Senegal and Zaire into Gabon. The transfer induced a deficiency in their trypanotolerance. Isometamidium chloride and diminazen aceturate were used. Key words : N'Dama cattle - Importation - Trypanocidal drug - Prophylaxis - Trypanotolerance - Gabon. bovins ne peuvent pas encore évoluer sans chimioprophylaxie antitrypanosomienne, car non seulement la trypanosomose reste à craindre, mais également d'autres maladies (cowdriose et anaplasmose) qui pourraient se révéler conjointement à l'effet immunosuppressif des trypanosomes (6). Des contrôles annuels permettront de déterminer si le programme de chimioprévention peut être allégé et si les bovins peuvent se passer complètement de traitement trypanocide.

DEHOUX (J.P.). Quemoprofilaxis antitripanosomosis de bovinos N'Dama importados de Senegambia y de Zaïre en Gabon. Revue Elev. Méd. vét. Pays trop., 1990, 43 (3): 337-341

Se estableció un programa de quimioprofilaxis antitripanosomosis, asociando aceturato de diminazeno $\mathbf{y}$ el cloruro de isometamidium, para bovinos de raza N'Dama importados recientemente del Senegal, de Gambia y de Zaire porque el traslado de dichos animales ocasionaba la desaparición de su tripanotolerancia. Palabras claves : Bovino N'Dama - Importación - Tripanocida - Profilaxis - Tripanotolerancia - Gabon.

\section{BIBLIOGRAPHIE}

1. AUTHIÉ (E.). Mise en évidence d'une résistance aux trypanocides parmi des souches de Trypanosoma congolense récemment isolées au Burkina. Revue Élev. Méd. vét. Pays trop., 1984, 37 (n spécial) : 219-225.

2. BOURN (D.). The successful use of work oxen in agricultural development of tsetse infested land in Ethiopia. Trop. Anim. Hlth Prod., 1978, 10: 191-203.

3. CAMUS (E.). Note sur un essai de traitement trypanocide pour lutter contre la primo-infection chez des veaux Baoulé. Revue Élev. Méd. vét. Pays trop., 1980, 33 (3) : 289-293.

4. CUNNINGHAM (M.P.). Vaccination du bétail contre les trypanosomes par l'infection et le traitement. In: 11 th meeting ISCTR, Nairobi, Kenya, 31 September-4 Novembcr 1966. Lagos, OAU-STRC, 1966. P. 65-67 (Publ. $\mathrm{n}^{\circ} 100$ ).

5. FINELLE (P.). Chimiothérapie et chimioprévention de la trypanosomiase animale, acquisitions récentes et situation actuelle. Cah. Méd. vét., 1973, 42 (5): 215-226.

6. HOMES (P.), MAMMO (E.). Immunosuppression in bovine trypanosomiasis. Vet. Rec., 1974, 95: 86-87.

7. ILCA. The African trypanotolerant livestock network. Indications from results, 1983-1985. Addis Abeba, ILCA, 1986

8. ILRAD. Rapport annuel du laboratoire international de recherches sur les maladies animales. Nairobi, ILRAD, 1986.

9. KAGERUKA (P.), MORTELMANS (J.), JOCHEMS (M.), AERTS (N.). Trypanosomiase du bétail trypanotolérant au Zaïre. Recherches parasitologiques et sérologiques dans un élevage nouvellement implanté. In: 18th meeting ISCTRC, Harare, Zimbabwe, 1985. Nairobi, OAU-STRC, 1985. P. 147-154 (Publ. $\mathrm{n}^{\circ} 113$ )

10. MURRAY (M.), MORRISON (W.I.), MURRAY (P.K.), CLIFFORD (D.J.), TRAIL (J.C.M.). La trypanotolérance Revue mond. Zootech., 1979, 31: 2-12.

11. PINDER (M.), AUTHIE (E.). The appearance of isometamidium resistant Trypanosoma congolense in West Africa Acta trop., 1984, 42:247-252

12. RÜCHEL (W.M.). Chemoprophylaxis of bovine trypanosomiasis. Eschborn, GTZ, 1975.

13. STARKEY (P.H.). Les bovins N'Dama, race trypanotolérante productive. Revue mond. Zootech., 1984, $50:$ 2-15. 
14. TACHER (G.). Utilisation de médicaments liée au développement de l'élevage dans les zones à glossines. Revue mond. Zootech., 1982, $44: 30-35$.

15. TOURE (S.M.), HOSTE (C.H.). Bétail trypanotolérant et trypanotolérance. Revue des connaissances. Bull. Séanc. Acad. r. Sci. Outre Mer, 1987, $32: 367-411$.

16. WHITELAW (D.D.), BELL (I.R.), HOLMES (P.H.), MOTOE (S.K.), HIRUMI (H.), URQHART (G.M.), MURRAY (M.). Isometamidium chloride prophylaxis against Trypanosoma congolense challenge and the development of immune responses in Boran cattle. Vet. Rec., 1986, 118 : 722-726.

17. WILSON (A.J.), PARIS (J.), LUCKINS (A.G.), DAR (F.K.), GRAY (A.R.). Observations on a herd of beef cattle maintained in a tsetse area. II. Assessment of the development of immunity in association with trypanocidal drug treatment. Trop. Anim. Hlth Prod., 1976, $8: 1-12$. 